\title{
Structural response of different Lewis number premixed flames interacting with a toroidal vortex
}

\author{
Chaimae Bariki ${ }^{\mathrm{a}}$, Fabien Halter ${ }^{\mathrm{a}, *}$, Fabien Thiesset ${ }^{\mathrm{a}}$, Christian Chauveau ${ }^{\mathrm{a}}$, Iskender Gökalp ${ }^{\mathrm{a}}$, Ketana Teav ${ }^{\mathrm{b}}$, \\ Sina Kheirkhah ${ }^{\mathrm{c}}$, Adam M. Steinberg ${ }^{\mathrm{b}}$ \\ ${ }^{a}$ ICARE-CNRS, Avenue de la Recherche Scientifique, Orléans Cedex2 45072, France \\ ${ }^{b}$ Institute for Aerospace Studies, University of Toronto, 4925 Dufferin Street, Toronto, Ontario M3H 5T6, Canada \\ ${ }^{c}$ School of Engineering, The University of British Columbia, 1137 Alumni Avenue, Kelowna, British Columbia, V1V1V7, Canada
}

\begin{abstract}
Simultaneous measurements of temperature, $\mathrm{CH}^{*}$ and $\mathrm{OH}^{*}$ chemiluminescent species are carried out to explore the impact of stretch rate and curvature on the structure of premixed flames. The configuration of an initially flat premixed flame interacting with a toroidal vortex is selected for the present study and reasons for this choice are discussed. Lewis number effects are assessed by comparing methane and propane flames. It is emphasized that the flame structure experiences very strong variations. In particular, the flame is shrunk (broadened) in the initial (final) period of the interaction with the vortex where strain rate (curvature) contribution of the stretch rate is predominant. By further analysing independently the thickness of the preheat and reaction zones, it is shown that for propane flames, not only the former but also the latter is significantly altered in zones where the flame curvature is negative. Changes in the reaction zone properties are further emphasized using $\mathrm{CH}^{*}$ and $\mathrm{OH}^{*}$ radicals. It is demonstrated that higher thermal diffusivity plays a significant role around curved regions, in which the enhanced diffusion of heat leads to a strong increase of $\mathrm{CH}^{*}$ compared to $\mathrm{OH}^{*}$ intensity. As an overall conclusion, this study suggests that it would be interesting to reassess the internal flame structure at lower and moderate Karlovitz numbers since changes might appear for a moderate vortex intensity with typical size much larger than the flame thickness.
\end{abstract}

Keywords:

Flame-vortex interaction, Laminar premixed flames, Curvature/stretch effects, Flame front thinning/thickening, Chemiluminescence

\footnotetext{
${ }^{*}$ Corresponding author: Halter)

Email address: fabien.halter@cnrs-orleans.fr (Fabien 


\section{Introduction}

When a premixed flame evolves in a non-uniform flow field, e.g. a turbulent flame, its geometry (flame surface area, curvature), kinematics (flame displacement and consumption speed), structure (thermal, preheat, and reaction zone thicknesses) and thermo-chemistry (species and temperature transport/production) can be altered. These flame features are coupled in a way that remains only partially understood, and hence a closed set of equations that accurately describe premixed flame behavior remains a research objective. Notwithstanding the complex nature of the fully turbulent problem, simplified - though insightful - configurations can yield insight regarding the elementary, leading order mechanisms at play in real turbulent premixed flames. From this perspective, the configuration of a single vortex interacting with an initially flat premixed flame is well-suited. In our opinion, this flow situation is equally relevant for fundamental purposes as steady laminar stretched flames or turbulent flames embedded in homogeneous isotropic turbulence.

Indeed, the literature demonstrates that flame/vortex interactions (FVI) have significantly helped in better understanding the effect of vortex strength on flame stretch and quenching [1, 2]. Such studies have led to the development of the so-called efficiency functions [3-6], which remain widely employed in Large Eddy Simulations (LES) of turbulent premixed combustion. Time transient effects [7-9] have also been evidenced using FVIs. More recently, Thiesset et al. [10] provided the first experimental confirmation of the asymptotic theory of Refs. [11, 12], revealing that flame speeds are unequally altered by flame curvature and hydrodynamic straining. Hence, two Markstein numbers should be used, one characterizing the dependence of flame speed to strain, the other to curvature. This demonstrates the utility of simultaneously observing strain and curvature in the same system; such results could not be obtained in, e.g, spherically expanding flames or steady stagnation point flames.

However, one aspect of FVI that has received less attention is the effect of vortex-induced strain and curvature on the flame structure and thermo-chemistry. The present study aims to fill this gap by experimentally determining the thermal structure and some indicators of diffusion and chemistry during FVI. The general goal is to use FVI to emphasize the effect of differential diffusion, i.e. the imbalance between thermal and molecular diffusivities as defined by the Lewis number ( $L e=\alpha / D_{d}$, where $\alpha$ is the thermal diffusivity and $D_{d}$ is the molecular diffusivity of the limiting reactant), in a 100 curved and strained flame.

Previous fundamental studies on differential diffusion effects have focused on e.g. the tip of a Bunsen flame [13-16]. This zone provides a convenient configuration to examine distortions between the temperature and species path-lines. Law et al. [13] and Mizomoto et al. [14] performed experiments using different fuels (e.g. methane, propane, and hydrogen) to demonstrate that negatively curved (concave towards the reactants) nonunity Lewis number flames exhibit changes not only in the internal flame structure, but also the flame burning rate. This result was later supported by DNS studies of Haworth and Poinsot [17] and Rutland and Trouvé [18], which considered a freely propagating premixed flame in homogeneous isotropic turbulence using single-step Arrhenius kinetics chemistry. They showed that negatively curved flame elements tend to have lower local flame speeds for $L e<1$, whereas the correlation is opposite for $L e>1$. Fewer studies have directly examined the internal flame structure of such flames. In DNS of equidiffusive flames (i.e. $L e=1$ ), Poinsot et al. [15] found that the preheat zone thickness increases in regions of negative curvature, while the reaction zone remains unaffected. However, there remains a knowledge gap regarding the internal flame structure for $L e \neq 1$.

The present study focuses on understanding the flame thermal structure and chemistry by carrying out simultaneous laser Rayleigh scattering thermometry, $\mathrm{OH}^{*}$ chemiluminescence, and $\mathrm{CH}^{*}$ chemiluminescence measurements in a FVI configuration. The impact of differential diffusion is assessed by comparing a stoichiometric methane flame $(L e \approx 1)$ and a slightly lean propane flame $(L e>1)$, while keeping the vortex characteristics relative to the flame constant. That is the ratio between the vortex rotational velocity and laminar flames, as well as the ratio between vortex size and flame thickness are held nearly constant in this study. Note that the case of $L e<1$ is not investigated here because these flames exhibit cellular thermodiffusive instabilities that alter the initial conditions for the FVI.

\section{Experimental set-up}

\subsection{Apparatus and diagnostic configuration}

Experiments were performed at the University of Toronto, using the FVI burner from Refs. [6, 10]. A flat premixed flame is stabilized in a single jet stagnation flow configuration before interacting with a toroidal vortex of prescribed strength. Premixed fuel and air are first laminarized using a grid to prevent turbulent structures from being convected in the burner. The reactive 
mixture then passes through a converging section hav- 137 ing an exit diameter of $15 \mathrm{~mm}$. The velocity profile at ${ }_{138}$ the exit of the contraction is nearly top hat. A burner ${ }_{139}$ to stagnation plate distance of $25 \mathrm{~mm}$ was chosen in or- 140 der to minimize the tangential strain rate before the FVI, ${ }_{141}$ while ensuring that the flame is repeatably stabilized. A 142 laminar coaxial co-flow of nitrogen is used to avoid the ${ }_{143}$ influence of external perturbations.

The toroidal vortex generator consists of a $2 \mathrm{~mm}$ tube 145 located on the centerline and ending $35 \mathrm{~mm}$ upstream 146 the burner exit. The vortex is generated by applying 147 a sudden pressure discharge of reactive mixture at the ${ }_{148}$ same equivalence ratio as the main flow. For this pur- 149 pose, the tube from which the vortex emanates is con- 150 nected to a pressurized tank via an electrovalve, which ${ }_{151}$ enables to control the vortex strength. Caution was ${ }_{152}$ taken to ensure that the vortex had identical composi- ${ }_{153}$ tion as the jet flow. This is done to avoid any discon- 154 tinuities or chemiluminescence signal variations due to 155 inhomogeneous equivalence ratio [19].

Measurements consisted of simultaneous 2D laser ${ }_{157}$ Rayleigh scattering (LRS) thermometry and chemilu- ${ }_{158}$ minescence (CL) measurements of $\mathrm{CH}^{*}$ and $\mathrm{OH}^{*}$. The ${ }_{159}$ setup is illustrated in Fig. 1 .

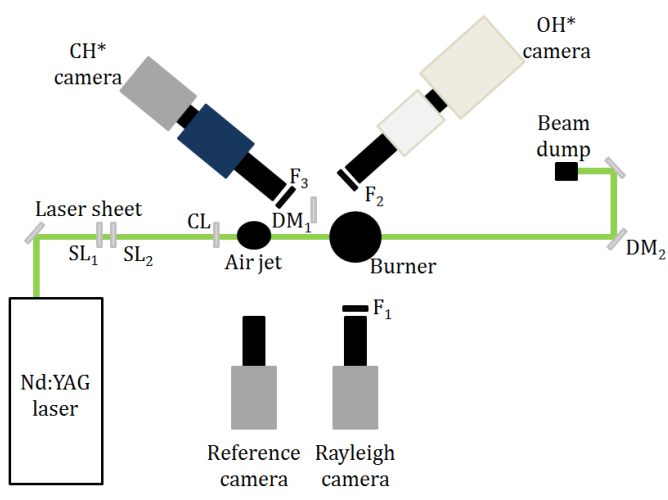

Figure 1: Optical configuration. CL: cylindrical lens, $\mathrm{DM}_{x}$ : dichroic mirror, $\mathrm{SL}_{x}$ : spherical lens, $\mathrm{F}_{x}$ : bandpass filter.

ity and compile phase-resolved statistics. Note that the $\mathrm{OH}^{*} \mathrm{CL}$ images were obtained at $10 \mathrm{kHz}$ repetition rate, and hence provide the complete interaction sequence for each vortex passage.

The LRS systems consisted of a $532 \mathrm{~nm} \mathrm{Nd:YAG}$ laser with a typical energy of $430 \mathrm{~mJ} /$ pulse (Spectra Physics Lab 170) and a sCMOS camera (Andor Zyla), operating at full resolution $\left(2160 \times 2560\right.$ pixels $\left.^{2}\right)$ and with a $10 \mu$ s exposure. The camera was fitted with a commercial lens (Tamron, $f / \#=3.5, f=180 \mathrm{~mm}$ ) and a $532 \pm 10 \mathrm{~nm}$ interference filter $\left(\mathrm{F}_{1}\right)$. In order to account for shot-to-shot laser power and profile variations, a reference sCMOS camera (Andor $\mathrm{Neo}$ ) was used to record a jet of clean air simultaneously with each burner LRS image. The laser sheet was formed using two spherical lenses $\left(f_{S L_{1}}=-100 \mathrm{~mm}, f_{S L_{2}}=150 \mathrm{~mm}\right)$ and a cylindrical lens $\left(f_{C L}=750 \mathrm{~mm}\right)$, resulting in a beam waist of $80 \mu \mathrm{m}$ and a height of $12 \mathrm{~mm}$.

The flame luminosity is split by a dichroic mirror $\left(\mathrm{DM}_{1}\right)$, which reflects the ultraviolet light from $\mathrm{OH}^{*}$ and transmits the visible light from $\mathrm{CH}^{*}$. The $\mathrm{OH}^{*} \mathrm{CL}$ system consists of a high-speed camera (Photron SA-Z, $10 \mathrm{kHz}, 1024 \times 1024$ pixels $^{2}$ ), image intensifier (Invisible Vision UVi, gate $90 \mu \mathrm{s}$ ), UV lens (Nikon, $f / \#=4.5$, $f=105 \mathrm{~mm})$, and bandpass filter $\left(\mathrm{F}_{2}\right.$, center wavelength $310 \mathrm{~nm}$, bandwidth $10 \mathrm{~nm})$. The $\mathrm{CH}^{*}$ system consists of a sCMOS camera (Andor Zyla, $2160 \times 2560$ pixels ${ }^{2}$ ), intensifier (LaVision IRO, gate $90 \mu \mathrm{s}$ ), camera lens (Tamron, $f / \#=3.5, f=180 \mathrm{~mm}$ ), and bandpass filter $\left(\mathrm{F}_{3}\right.$, center wavelength $430 \mathrm{~nm}$, bandwidth $10 \mathrm{~nm}$ ). The cameras images were aligned using a dot target. The projected pixel resolutions are 7.7, 16.7 and $43.3 \mu \mathrm{m} / \mathrm{px}$ for Rayleigh, $\mathrm{CH}^{*}$, and $\mathrm{OH}^{*}$ chemiluminescence techniques respectively. Note that all the cameras were corrected for their corresponding whitefield responses and backgrounds.

The chemiluminescence measurement technique provides a line-of-sight integrated field. Since the configuration is highly symmetric, Abel deconvolution was applied to obtain planar images of $\mathrm{CH}^{*}$ and $\mathrm{OH}^{*}$ at the mid-plane. The BAsis Set Expansion (BASEX) method was used [20]. Negligible sensitivity was found to the input or parameters of the BASEX algorithm.

\subsection{Selected experimental conditions}

Stoichiometric $(\phi=1)$ methane/air and lean $(\phi=$ 0.9) propane/air mixtures were selected to study the flame response to curvature and thermo-diffusive effects. The vortex parameters, i.e. the rotational velocity $U_{\theta}=0.9 \mathrm{~m} / \mathrm{s}$, convection velocity $U_{c}=0.35 \mathrm{~m} / \mathrm{s}$, and core-to-core distance $R_{v}=4 \mathrm{~mm}$, were determined just before the vortex starts interacting with the flame 
Table 1: Experimental conditions and key parameters: adiabatic flame temperature $\left(T_{p}\right)$, vortex core-to-core distance $\left(R_{v}\right)$, thermal flame thickness $\left(\delta_{L}^{0}\right)$, vortex rotational velocity $\left(U_{\theta}\right)$, laminar flame speed $\left(S_{L}^{0}\right)$, and effective Lewis number $L e_{e f f}$.

\begin{tabular}{|c|c|c|c|c|c|c|}
\hline Case & Fuel & $\phi$ & $T_{p}$ & $R_{v} / \delta_{L}^{0}$ & $U_{\theta} / S_{L}^{0}$ & $L e_{e f f}$ \\
\hline M10 & $\mathrm{CH}_{4}$ & 1.0 & 2200 & 9.6 & 2.5 & 1.05 \\
\hline P09 & $\mathrm{C}_{3} \mathrm{H}_{8}$ & 0.9 & 2157 & 11.3 & 2.45 & 1.5 \\
\hline
\end{tabular}

using previous measurements [6, 10]. Flame character- 220 istics are calculated for a 1D stagnation laminar flame ${ }^{221}$ configuration, performed using the PREMIX module in 222 CHEMKIN-II. The GRI-Mech 3.0 [21] chemical mech- ${ }^{223}$ anism was used for the methane flame and ARAMCO- 224 Mech 2.0 [22] for the propane flames. The flame ther- 225 mal thickness is defined as $\delta_{L}^{0}=|\nabla c|_{\text {max }}^{-1}$, where the 226 progress variable $c=\left(T-T_{r}\right) /\left(T_{p}-T_{r}\right), T_{r}$ is the reac- 227 tant temperature, and $T_{p}$ is the adiabatic flame temperature. $T_{p}$ was found to be nearly the same for the two selected cases meaning that differential thermal expan- ${ }_{228}$ sion is negligible.

The effective Lewis numbers $\left(L e_{e f f}\right)$ are evaluated using the methodology of Bechtold et al. [11]. Stoichiometric methane/air mixture is thermo-diffusionally neutral (i.e. $L e_{e f f} \approx 1$ ), whilst the lean propane flame ${ }^{230}$ is thermo-diffusionally stable $\left(L e_{e f f} \approx 1.5\right)$. Note that ${ }^{231}$ these cases have nearly identical $U_{\theta} / S_{L}$, meaning that ${ }^{232}$ differential flow effects are eliminated and the Lewis ${ }^{233}$ number effect can be isolated. Table 1 outlines the key ${ }^{234}$ parameters of the two selected conditions.

\subsection{Temperature inversion methodology}

Instantaneous LRS images were first binned into $4 \times 4{ }^{238}$ windows in order to increase the signal-to-noise ratio, and then filtered using a $8 \times 8$ Wiener filter. The size of the filter was chosen in a way that it does not modify the flame front structure.

Assuming isobaric flow of an ideal gas, LRS signal ${ }^{243}$ can be inverted to temperature via

$$
T=T_{\text {ref }} \frac{I_{\text {ref }}-I_{B G}}{I_{\text {flame }}-I_{B G}} \frac{(\partial \sigma / \partial \Omega)_{\text {mix }}}{(\partial \sigma / \partial \Omega)_{\text {mix }, \text { ref }}}
$$

flese and Iref represent the Rayleigh scattered 240 light from the flame and a calibration image of fresh gases at a known temperature, respectively. $I_{B G}$ is the ${ }^{25}$ background intensity, which consists of the dark field of 252 the camera and the laboratory background noise. The ${ }_{253}$ differential Rayleigh scattering cross-section $(\partial \sigma / \partial \Omega){ }_{254}$ of the local gas is the mole-fraction weighted sum of 255 the differential cross-sections of the individual species. Individual molecular cross-sections were calculated as

$$
\sigma_{i}=\frac{4 \pi^{2}}{\lambda^{4}}\left(\frac{n-1}{N_{0}}\right)^{2}\left(\frac{3}{3-4 \rho_{p}}\right)\left(1+\rho_{p}\right)
$$

where $\lambda$ is the laser wavelength, $n$ is the index of refraction, $N_{0}$ is the Loschmidt number and $\rho_{p}$ is the depolarization ratio [23-25]. The variation of composition with temperature was assumed to follow that of a planar laminar flame, computed as described above. The temperature uncertainty was calculated based on the Poisson noise since it is dominated by the shot-noise, and is equal to $5 \mathrm{~K}$ and $50 \mathrm{~K}$ in the reactants and products, respectively.

The resultant temperature fields were converted to progress variable fields. These were used to calculate the flame thermal thickness from the progress variable gradient.

\section{Results and Discussion}

\subsection{Flame interacting with a toroidal vortex}

A time sequence of temperature fields from the FVI in the propane/air flame is shown in Fig. 2. Two somewhat distinct time periods are apparent, one in which the vortex modifies the flame and one in which the flame returns to its unperturbed state. At $t=0 \mathrm{~ms}$, the flame was almost flat, with a slight positive curvature due to the weak wake from the vortex-generating tube. For $0<t \lesssim 4.5 \mathrm{~ms}$, the toroidal vortex was injected, convected to the flame, and distorted the flame front. The vortex did not cause local extinction, and the flame returned to its original shape over $t \gtrsim 4.5 \mathrm{~ms}$. This second time period is characterized by very high negative curvatures and is driven by the consumption velocity.

The normalized rate-of-change in flame surface area, $A$, constitutes the total stretch rate. The stretch-rate can be decomposed into two contributions, one due to tangential strain and one to the propagation of curved flame elements. The time evolution of flame area is displayed in Fig. 3 for both cases. The flame response to the FVI was almost identical during the initial phase of the FVI, until $t \approx 3.8 \mathrm{~ms}$, which is dominated by vortex-induced strain causing increased $A$. However, significant differences occurred between the $L e_{\text {eff }} \approx 1$ methane/air flame and $L e_{e f f}>1$ propane/air flame during the second phase of the FVI, in which the highly curved flame returned to its original configuration. 


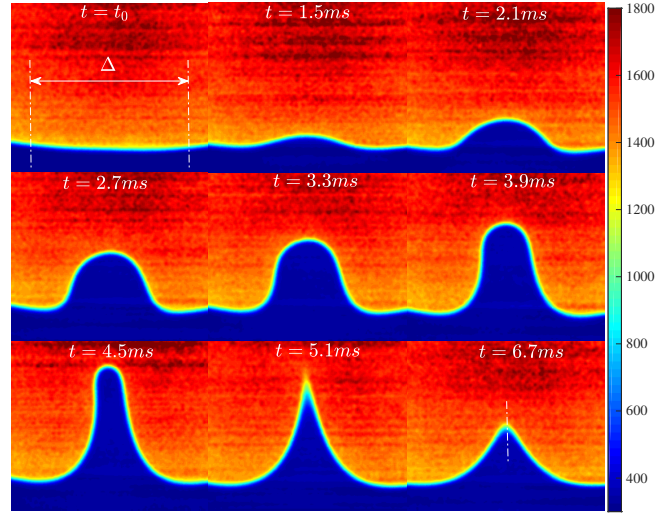

Figure 2: Time sequence of temperature field for propane flame at $\phi 286$ $=0.9$. $\Delta$ is the width domain. The centerline at $6.7 \mathrm{~ms}$ is where the 287 axial profiles will be extracted.

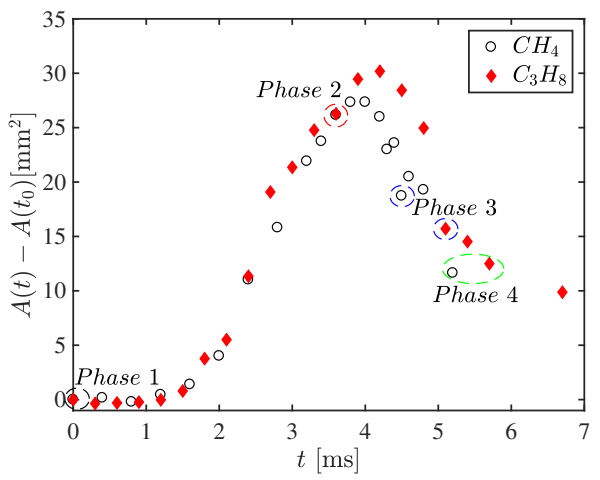

Figure 3: Time evolution of flame area $A(t)$ for P09 (red diamonds) and M10 (black circles) cases. The selected phases to be compared ${ }^{303}$ are circled, characterized by approximately the same curvature at the 304 flame tip.

To investigate this further, the total flame curvature, 309 $\kappa_{m}$, was calculated as the average of the in-plane curva- 310 ture $\kappa_{1}=r^{\prime} h^{\prime \prime}-r^{\prime \prime} h^{\prime}$ and the contribution in the circum- 311 ferential direction $\kappa_{2}=h^{\prime} / r$. Here, $r$ and $h$ are the ra- 312 dial and the streamwise spatial flame front coordinates, 313 respectively, indexed against the curvilinear abscissa $s, 314$ and $(\cdot)^{\prime}$ is a derivative with respect to $s$. Curvature was 315 extracted along the $c=0.7$ contour, which is represen- 316 tative of the reaction zone.

To demonstrate the effects of curvature and differen- 318 tial diffusion on the inner flame structure, four phases 319 were selected from each flame for which the minimum 320 radius of curvature (i.e. along the burner centerline) was 321 nearly identical between the fuels. These phases are cir- 322 cled in Fig. 3. The first two phases correspond to the ${ }_{323}$ early stage of FVI where strain rate is predominant over ${ }_{324}$ curvature, whereas the two last phases correspond to the second part of FVI where the flame tip is extremely curved. During the early stage, the equal curvatures occurred at the same time in the vortex passage. However, the curvature of the propane flame lagged that of the methane flame by approximately $0.5 \mathrm{~ms}$ during the later stage of the FVI. This lag could be explained by the initial state of propane flame that is less positively curved than methane flame. Recall that at steady state, the flames are slightly convex towards the unburned reactants due to the vortex generator that creates a small velocity deficit in its wake [10].

The inner flame structure during the FVI is examined in Fig. 4 by the variation of $|\nabla c|$ with $c$ at the flame centerline $(r=0)$ for these phases. This parameter is inversely related to the thermal flame thickness. Note that for each fuel, each phase has been normalized by the corresponding maximum value of $|\nabla c|$ when the flame is flat, i.e. at Phase 1 before the interaction takes place. The first phase illustrates the initial inner flame structure affected only by the relatively weak straining from the stagnation flow. The curves for Phase 1 are relatively symmetric around $c=0.5$.

Phase 2 occurred during the upward movement of the flame as it was strained and wrinkled by the vortex. At this phase, $|\nabla c|$ exhibits greater values compared to the initial phase, indicating a relative thinning of 30 and $40 \%$ for methane and propane flames, respectively. In Ref. [26], it was demonstrated that equidiffusive flame structure remains insensitive to strain rate using a counterflow configuration. On the other hand, Driscoll [27] states in his review that a positively strained lean methane flame becomes thinner, whereas a lean propane flame becomes thicker. The result presented here for FVI brings new insights.

For a highly negatively curved shape at Phase 3, a prominent decrease of $|\nabla c|$ is identified, meaning that the flame front is broadened. Furthermore, the profile becomes asymmetric, with elevated $|\nabla c|$ for $0.45<c<$ 1 in the methane flame and a similar pattern, though with lower amplitude, in the propane flame. When the curvature decreases (Phase 4), the thickening diminishes for both fuels but faster for methane.

Changes to the internal flame structure during FVI are further elucidated by decomposing the flame front into two regions, one approximately describing the thickness of the preheat zone and the other the reaction zone thickness. The preheat zone thickness along the length of the flame $\left(\delta_{\text {pre }}\right)$ is characterized by the minimum distance between the $c=0.1$ and $c=0.7$ iso-contours at the same phases used in Fig. 4. In Fig. 5(a), this thickness is plotted versus the local flame curvature on the 


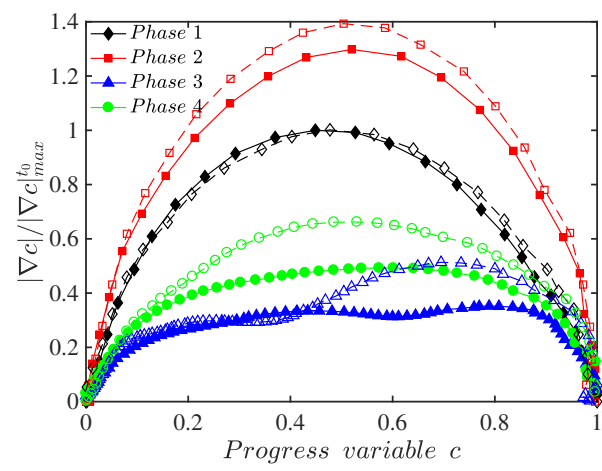

Figure 4: Normalized $|\nabla c|$ profiles versus progress variable $c$ at the flame centerline for different phases during FVI for P09 case (filled symbols) and M10 case (open symbols). $c=0.7$ iso-contour. For Phases 1 and 2, normalized curvatures are in the range $-0.3 \lesssim \kappa_{m} \cdot \delta_{L} \lesssim 0.2$ and the preheat zone thickness remains nearly constant. However, significant changes are observed for Phases 3 and 4 at locations where $\kappa_{m} \cdot \delta_{L} \lesssim-0.3$; the preheat zone can be thickened by nearly a factor of four. For highly negatively curved shapes (i.e. Phase 3 ), the methane preheat zone is more broadened compared to the propane flame. ${ }^{360}$ The preheat layer thickening in highly curved regions ${ }^{361}$ occurs near the burner centerline, whereas the weakly curved edges of the flame remain relatively unaffected ${ }^{362}$ by the FVI.

Because the most significant changes are observed in ${ }_{364}$ the highly curved region at the centerline, the reaction ${ }_{365}$ zone thickness is examined at this location as a function 366 of time. The reaction zone thickness $\delta_{\text {rec }}$ is defined as ${ }_{367}$ the distance between $c=0.7$ and $c=0.85$ at $r=0$ and ${ }_{368}$ shown versus the local curvature in Fig. 5.b). The time ${ }_{369}$ evolution manifests as first decreasing and then increas- 370 ing curvature values. For large negative curvatures, the ${ }_{371}$ reaction zone is strongly thickened for the $L e_{e f f}>1{ }_{372}$ propane/air flame, but only slightly thickened for the ${ }_{373}$ $L e_{e f f} \approx 1$ methane/air flame.

Note that Phase 2 and the early portion of the inter- 375 action correspond to times of high strain rate. However, ${ }_{376}$ the preheat and reaction zone thicknesses are only min- 377 imally altered during this time. Hence, the flame thick- 378 ness is much more sensitive to curvature than strain. 379 Moreover, for both the preheat and reaction zones, 380 structural changes are noticeable only when the radius 381 of curvature is smaller than twice the laminar flame ${ }_{382}$ thickness.

Previous conclusions pertain to the thermal structure 384 of the flame. We now turn our attention to some in- 385 dicators of the kinetic activity of the reaction zone by ${ }_{386}$

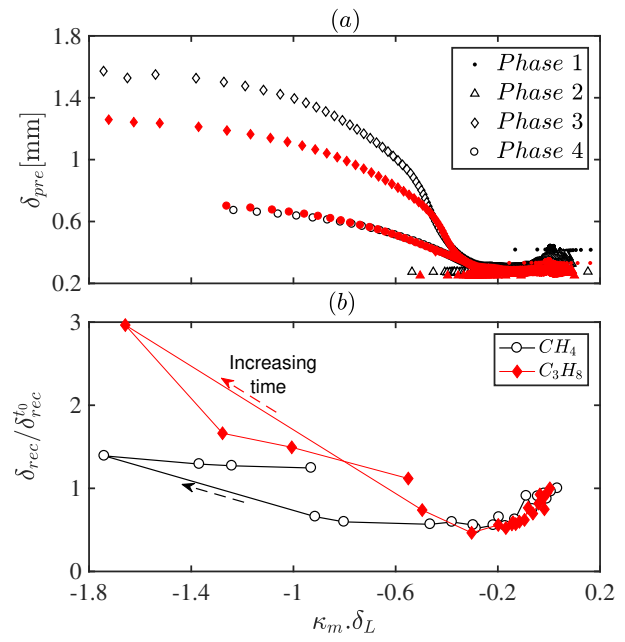

Figure 5: (a) Preheat zone thickness vs normalized curvature along the flame contour for four different phases (each phase is represented by a specific symbol). (b) Normalized reaction zone thickness evaluated at the flame tip during the whole FVI. Curvature is normalized with the laminar flame thermal thickness. Red (filled) and black (open) colors (symbols) represent $\mathrm{C}_{3} \mathrm{H}_{8} /$ air and $\mathrm{CH}_{4}$ /air mixtures. The arrow indicates the direction of increasing time.

analysing the chemiluminescence signals of $\mathrm{OH}^{*}$ and $\mathrm{CH}^{*}$.

\section{3. $\mathrm{CH}^{*}$ and $\mathrm{OH}^{*}$ measurements}

Given the above-noted changes to the reaction zone thickness in highly negatively curved flame segments, it is useful to further investigate the thermal and chemical processes occurring in this region. $\mathrm{CH}^{*}$ and $\mathrm{OH}^{*}$ are two reasonable metrics representing the reaction zone. However, meaningful interpretation of chemiluminescence measurements requires knowledge of the kinetics leading to the formation of the excited state. The reaction of ethynyl radical $\mathrm{C}_{2} \mathrm{H}$ with atomic oxygen is the dominant source of $\mathrm{CH}^{*}$ formation $\left(\mathrm{C}_{2} \mathrm{H}+\mathrm{O} \leftrightarrow\right.$ $\mathrm{CH}^{*}+\mathrm{CO}$ ) [28], whereas the emission intensity of $\mathrm{OH}^{*}$ is mainly proportional to the local concentration of $\mathrm{CH}$ and $\mathrm{O}_{2}\left(\mathrm{CH}+\mathrm{O}_{2} \leftrightarrow \mathrm{OH}^{*}+\mathrm{CO}\right)$ for hydrocarbon flames [29]. The ratio $\mathrm{OH}^{*} / \mathrm{CH}^{*}$ gives an indication of the balance of these two mechanisms.

Figure 6 displays Abel deconvoluted images of $\mathrm{CH}^{*}$, $\mathrm{OH}^{*}$ and $\mathrm{OH}^{*} / \mathrm{CH}^{*}$ for a curved propane flame at Phase 3. Elevated $\mathrm{CH}^{*}$ concentrations occurred at the flame tip, whereas the $\mathrm{OH}^{*}$ concentration was relatively constant along the flame; the $\mathrm{OH}^{*} / \mathrm{CH}^{*}$ ratio decreased near the flame tip. The relative decrease of $\mathrm{OH}^{*}$ compared to $\mathrm{CH}^{*}$ could be due to several factors. In Refs. [30, 31], it was found that $\mathrm{OH}^{*} / \mathrm{CH}^{*}$ ratio was inversely proportional to equivalence ratio for lean and stoichiometric 


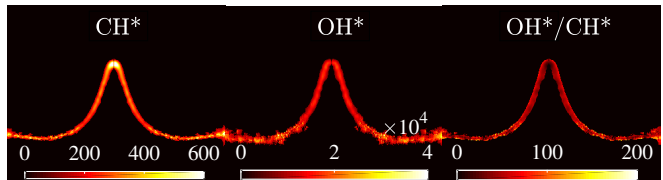

Figure 6: Snapshots of $\mathrm{CH}^{*}, \mathrm{OH}^{*}$ and $\mathrm{OH}^{*} / \mathrm{CH}^{*}$ (left to right) for a 435 curved propane flame at Phase 3.

flames. However, differential diffusion of propane and oxygen would be expected to result in leaner conditions at the flame tip, which is counter to the experimental results.

Recall that the lean propane flame is characterized by $L e_{\text {eff }}>1$, meaning that thermal diffusion is dominant compared to molecular diffusion. This suggests that the increase in $\mathrm{CH}^{*}$ intensity in regions of high negative curvature may be attributed to higher thermal diffusivity enhancing the formation of $\mathrm{CH}^{*}$ precursors. This was verified numerically using a counterflame configuration, which showed that preheating fresh reactants increases the concentration of $\mathrm{O}, \mathrm{C}_{2} \mathrm{H}$, and $\mathrm{CH}^{*}$. In contrast, the concentration of $\mathrm{OH}^{*}$ was relatively unchanged because the majority of $\mathrm{O}_{2}$ is consumed before reaching the $\mathrm{OH}^{*}$ layer. Hence, the increase in $\mathrm{CH}^{*}$ and decrease in $\mathrm{OH}^{*} / \mathrm{CH}^{*}$ near the flame tip is due to preferential thermal diffusion in this region.

To further quantify the effect of local curvature on chemiluminescence, $\mathrm{CH}^{*}$ and $\mathrm{OH}^{*}$ intensities were integrated normally to the $c=0.7$ iso-contour and then normalized by a reference value corresponding to the flat flame. These are plotted versus the local flame cur- 440 vature in Fig. 7. For the propane flame, both $\mathrm{CH}^{*}$ and $\mathrm{OH}^{*}$ were negatively correlated with curvature, though with much greater sensitivity for $\mathrm{CH}^{*}$ in the range ${ }_{442}$ $-0.6 \lesssim \kappa_{m} \cdot \delta_{L} \lesssim-0.2$. Beyond $\kappa_{m} \cdot \delta_{L} \lesssim-0.6$, neither ${ }_{443}$ $\mathrm{OH}^{*}$ nor $\mathrm{CH}^{*}$ were sensitive to curvature.

A similar behavior has been identified for the effect of 445 curvature on the local burning velocity [17, 18, 32]; neg- 446 atively curved flame segments have higher local flame 447 speeds for $L e_{e f f}>1$, whereas, the correlation is op- 448 posite for $L e_{e f f}<1$. The curve representing the local 449 burning velocity as a function of normalized curvature 450 for a $\phi=0.7$ propane flame, provided by Bell et al. ${ }_{451}$ [32], is also shown in Fig. 7. As can be seen from ${ }_{452}$ this figure, the slopes of the three curves are different. ${ }_{453}$ This means that $\mathrm{CH}^{*}$ and $\mathrm{OH}^{*}$ radicals are not directly ${ }_{454}$ correlated to local burning velocity or heat release rate 455 when the flame is curved. There might be other pa- 456 rameters to consider in order to deduce the right de- 457 pendence between chemiluminescent species and heat 458 release rate. Hence, $\mathrm{CH}^{*}$ and $\mathrm{OH}^{*}$ species can be inter- ${ }_{459}$ preted as qualitative indicators of heat release rate and cannot be used to quantify it.

Concerning the methane flame, it was found that $\mathrm{CH}^{*}$ and $\mathrm{OH}^{*}$ intensities are not strongly affected by curvature and remain nearly constant (see Fig. 7). One would expect that mixtures with $L e_{e f f} \approx 1$ will have such a behavior. It is important to note that intermediate species as atomic hydrogen and atomic oxygen may also have a significant impact, particularly for flames characterized by $L e<1$.

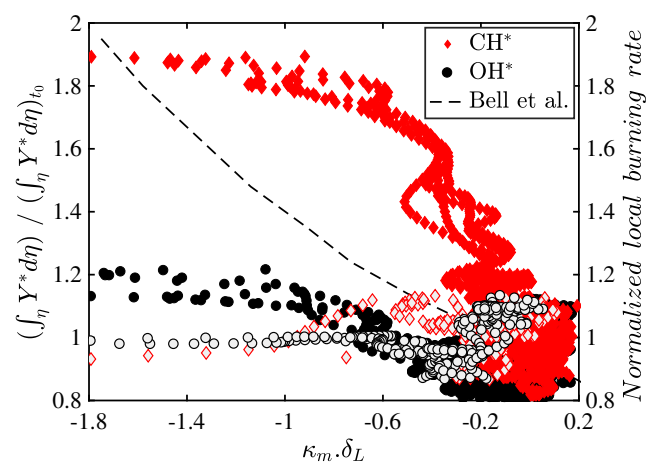

Figure 7: Normalized and integrated values of the $\mathrm{CH}^{*}$ (red symbols) and $\mathrm{OH}^{*}$ (black symbols) intensities along the flame contour vs the normalized curvature. Filled and open symbols stand for propane and methane flames respectively. Overlaid dashed curve is reproduced from the results presented in Bell et al. [32].

\section{Concluding remarks}

The influence of stretch and curvature on the structure of different Lewis number flames have been explored in the particular configuration of a flame/vortex interaction. The imbalance between thermal and reactant diffusion is evaluated by comparing a stoichiometric methane flame to a lean propane flame. Analysis of the thermal structure and the kinetic activity is undertaken on the basis of Rayleigh scattering together with $\mathrm{CH}^{*}$ and $\mathrm{OH}^{*}$ chemiluminescence measurements, respectively.

In the present case, the vortex intensity and size relative to the flame properties lies within the wrinkled flame regime of Refs. [1, 2]. Therefore, one should have expected only marginal structural variations of the flame zone. Moreover, it is often postulated that even though the preheat zone might be strongly influenced by hydrodynamic straining and curvature, the reaction zone is much more robust and experiences only marginal thickness variations. Our study demonstrates that these two 
statements are violated even at moderate vortex inten- 509 sity and large vortex size. Indeed, a significant thinning 510 (thickening) is observed during the first (second) period ${ }^{511}$ of the interaction where the strain rate (curvature) con- ${ }_{513}^{512}$ tribution to stretch dominates. This reinforces the need 514 for distinguishing strain and curvature effects as in Refs. ${ }^{515}$ [10-12] for the flame speeds. More importantly, by dif- ${ }^{516}$ ferentiating the flame zone into a preheat and reaction ${ }_{518}^{517}$ zone, it is clearly emphasized that the structure of the 519 reaction zone can be also profoundly altered. The thick- ${ }^{520}$ ening of the reaction was observed only when the flame ${ }^{521}$ radius of curvature was of the order of the flame thick- ${ }_{523}$ ness and for a non unity Lewis number flame. These ob- 524 servations are further confirmed by analysing $\mathrm{CH}^{*}$ and ${ }^{525}$ $\mathrm{OH}^{*}$, i.e. two reasonable markers of the reaction zone ${ }_{527}^{526}$ activity. We show that the reaction zone of a propane ${ }_{528}$ (methane) flame is significantly (marginally) sensitive ${ }^{529}$ to curvature.

As an overall conclusion, temperature fields together ${ }_{532}^{531}$ with the chemiluminescence signals of $\mathrm{CH}^{*}$ and $\mathrm{OH}^{*}{ }_{533}$ highlight that in contrast with common statements, a 534 moderate vortex intensity with typical size much larger ${ }^{535}$ than the flame thickness is sufficient to induce signif- ${ }_{537}^{536}$ icant changes in the flame structure. Although FVIs 538 should be interpreted as an intermediate situation be- ${ }^{539}$ tween stretched laminar and turbulent flames, the cur- 540 rent results suggest that the inner flame flame structure ${ }_{542}$ may be modified at lower Karlovitz numbers than clas- 543 sically expected.

\section{Acknowledgments}

The authors gratefully acknowledge the financial 550 support provided by the French Government Pro- ${ }^{551}$ gram "Investissements d'avenir" through the LABEX ${ }_{553}^{552}$ CAPRYSSES ANR-11-LABX-0006-01, NSERC ${ }_{554}^{553}$ Canada through Grant RGPIN 2017-06501, and the 555 Ontario Ministry of Research and Innovation Early ${ }^{556}$ Researcher Award program. We are also thankful to the ${ }^{557}$ CNRS and the University of Orléans.

\section{References}

[1] T. Poinsot, D. Veynante, S. Candel, J. Fluid Mech. 228 (1991) 561-606.

[2] W. L. Roberts, J. F. Driscoll, M. C. Drake, L. P. Goss, Combust. Flame 94 (1993) 58-69.

[3] O. Colin, F. Ducros, D. Veynante, T. Poinsot, Phys. Fluids 12 (2000) 1843-1863.

[4] F. Charlette, C. Meneveau, D. Veynante, Combust. Flame 131 (2002) 159-180.

[5] S. Bougrine, S. Richard, O. Colin, D. Veynante, Flow, Turbul. Combust. 93 (2014) 259-281.
[6] F. Thiesset, G. Maurice, F. Halter, N. Mazellier, C. Chauveau, I. Gökalp, Proc. Combust. Inst. 36 (2017) 1843-1851.

[7] J.-M. Samaniego, T. Mantel, Combust. Flame 118 (1999) 537556.

[8] T. Mantel, J.-M. Samaniego, Combust. Flame 118 (1999) 557582.

[9] C. Mueller, J. Driscoll, D. Reuss, M. Drake, Proc. Combust. Inst. 26 (1996) 347-355.

[10] F. Thiesset, F. Halter, C. Bariki, C. Lapeyre, C. Chauveau, I. Gökalp, L. Selle, T. Poinsot, J. Fluid Mech. 831 (2017) 618654.

[11] J. Bechtold, M. Matalon, Combust. Flame 127 (2001) 19061913.

[12] G. K. Giannakopoulos, A. Gatzoulis, C. E. Frouzakis, M. Matalon, A. G. Tomboulides, Combust. Flame 162 (2015) 12491264.

[13] C. Law, S. Ishizuka, P. Cho, Combust. Sci. Technol. 28 (1982) 89-96.

[14] M. Mizomoto, Y. Asaka, S. Ikai, C. Law, Proc. Combust. Inst. 20 (1985) 1933-1939.

[15] T. Poinsot, T. Echekki, M. Mungal, Combust. Sci. Technol. 81 (1992) 45-73.

[16] T. M. Vu, M. S. Cha, B. J. Lee, S. H. Chung, Combust. Flame 162 (2015) $1614-1621$.

[17] D. Haworth, T. Poinsot, J. Fluid Mech. 244 (1992) 405-436.

[18] C. Rutland, A. Trouvé, Combust. Flame 94 (1993) 41-57.

[19] C. M. Vagelopoulos, J. H. Frank, Proc. Combust. Inst. 30 (2005) 241-249.

[20] V. Dribinski, A. Ossadtchi, V. A. Mandelshtam, H. Reisler, Rev. Sci. Instrum. 73 (2002) 2634-2642.

[21] G. P. Smith, D. M. Golden, M. Frenklach, N. W. Moriarty, B. Eiteneer, M. Goldenberg, C. T. Bowman, R. K. Hanson, S. Song, W. C. Gardiner Jr, et al., Gas Research Inst. (1999).

[22] C.-W. Zhou, Y. Li, E. O'Connor, K. P. Somers, S. Thion, C. Keesee, O. Mathieu, E. L. Petersen, T. A. DeVerter, M. A. Oehlschlaeger, et al., Combust. Flame 167 (2016) 353-379.

[23] W. Gardiner, Y. Hidaka, T. Tanzawa, Combust. Flame 40 (1981) 213-219.

[24] J. Fielding, J. H. Frank, S. A. Kaiser, M. D. Smooke, M. B. Long, Proc. Combust. Inst. 29 (2002) 2703-2709.

[25] J. A. Sutton, J. F. Driscoll, Opt. Lett. 29 (2004) 2620-2622.

[26] C. Law, C. Sung, G. Yu, R. Axelbaum, Combust. Flame 98 (1994) 139-154.

[27] J. F. Driscoll, Prog. Energy Combust. Sci. 34 (2008) 91-134

[28] K. Devriendt, J. Peeters, J. Phys. Chem. A 101 (1997) 25462551.

[29] S. Carl, M. Van Poppel, J. Peeters, J. Phys. Chem. A 107 (2003) 11001-11007.

[30] J. Kojima, Y. Ikeda, T. Nakajima, Combust. Flame 140 (2005) 34-45.

[31] M. Orain, Y. Hardalupas, CR Mecanique 338 (2010) 241-254.

[32] J. B. Bell, R. K. Cheng, M. S. Day, I. G. Shepherd, Proc. Combust. Inst. 31 (2007) 1309-1317. 